\title{
On Ramanujan congruences between special values of Hecke and Dirichlet $L$-functions
}

\author{
by \\ P. Guerzhoy (Mannheim)
}

Introduction. The Fourier coefficients of modular forms of even integer weight on the full modular group $\mathrm{SL}(2, \mathbb{Z})$ can by now be considered classical in number theory. Among their intriguing properties appears a remarkable congruence of Ramanujan $\tau(n) \equiv \sigma_{11}(n)$ mod 691 . Plenty of excellent works, such as [9], [10], [12] and [13], that appeared in the last two decades were devoted to various generalisations of this congruence.

In the present paper we consider how this congruence and its higher weight analogues can be carried over to congruences between special values of $L$-functions of modular forms. The first two examples of this phenomenon appeared in the works of N. Koblitz [6] and [7]. In this paper we present a general construction which is based on the classical Rankin's method. This construction yields Koblitz type congruences in an arbitrary weight, provided an additional hypothesis (see H1 below) is satisfied in that weight. Computer calculations show that $\mathrm{H} 1$ is satisfied for all weights less than or equal to 34 . We give a heuristic argument that H1 is satisfied frequently.

The philosophic foundation of the results of this type is explained in [6]. We will briefly recall it. If we deal with an arithmetic object to which one can associate a motivated $L$-function, then we can expect that congruences between objects induce, after removing some transcendental factors, congruences modulo the same ideals between the special values of their $L$-functions. This phenomenon becomes less striking if we take into account that these special values are up to a common factor algebraic numbers. The present construction can be considered as an attempt to prove some assertions along these lines when the "arithmetic objects" are modular forms and the "congruences" are Ramanujan type congruences.

1991 Mathematics Subject Classification: Primary 10D23; Secondary 10D24, 10D12. Supported by Minerva fellowship. 
The content of the paper is as follows. In the first section we state the main result and consider some corollaries. In particular, we obtain two assertions of [6] and [7] as special cases of our theorem. The second section is devoted to the proof of this result.

The author is very grateful to Prof. A. A. Panchishkin (Institut Fourier, France) who taught him the Rankin's convolution method. The author is very indebted to Prof. B. Datskovsky (Temple University, USA) because this paper was born in regular and fruitful conversations with him without which it would have never been written. The author is also grateful to Prof. D. Zagier (Max-Planck-Institut für Mathematik, Bonn) for a useful communication.

Finally, the author expresses his gratitude to the referee for his hints to improve the presentation of the article.

Notations. For an even integer $k \geq 12$ we denote by $S_{k}$ the space of cusp forms of weight $k$ for the full modular group $\mathrm{SL}_{2}(\mathbb{Z})$. We call a cusp form primitive if it is a common eigenfunction of all Hecke operators, normalised so that the first coefficient $a(1)$ of its Fourier expansion

$$
f(\tau)=\sum_{n \geq 1} a(n) q^{n}, \quad q=\exp (2 \pi i \tau),
$$

is equal to 1 . This definition agrees formally with the standard one which is applicable to modular forms for congruence subgroups. We denote by $G_{k}$ the Eisenstein series of weight $k$ normalised as usual:

$$
G_{k}=-B_{k} /(2 k)+\sum_{n \geq 1} \sigma_{k-1}(n) q^{n} .
$$

The canonical Hecke $L$-series

$$
L_{f}(s, \psi)=\sum_{n \geq 1} \psi(n) a(n) n^{-s}
$$

is associated with each cusp form $f$ and Dirichlet character $\psi$. These series converge for $\Re(s) \gg 0$ and have a meromorphic continuation to the whole complex plane.

For any periodic function $\psi: \mathbb{Z} \rightarrow \mathbb{C}$ we denote by $L(s, \psi)$ the (generalised) Dirichlet $L$-series

$$
L(s, \psi)=\sum_{n \geq 1} \psi(n) n^{-s} .
$$

The symbol $\psi^{0}$ will denote the trivial Dirichlet character modulo 1 , and we will write simply $L_{f}(s)$ and $\zeta(s)$ instead of $L_{f}\left(s, \psi^{0}\right)$ and $L\left(s, \psi^{0}\right)$. $G(\psi)$ will denote the Gauss sum associated with the Dirichlet character $\psi$. 
The symbol $\sigma_{s}(d, \psi, \chi)$ for natural numbers $s$ and $d$ and Dirichlet characters $\psi$ and $\chi$ denotes the arithmetic function

$$
\sigma_{s}(d, \psi, \chi)=\sum_{x \mid d} x^{s} \psi(x) \chi(d / x) .
$$

1. Let $b_{k}$ denote the numerator of the rational number $B_{k} /(2 k)$ written in the lowest terms where $B_{k}$ is the Bernoulli number, $B_{k}=-k \zeta(1-k)$.

Proposition 1. There exists a cusp form $g$ with integral Fourier coefficients such that $g \equiv G_{k} \bmod b_{k}$.

To prove Proposition 1 notice that $E_{4}=-240 G_{4}$ and $E_{6}=504 G_{6}$ have integral Fourier coefficients and constant term equal to 1 . Using $E_{4}$ and $E_{6}$ we can construct a modular form $h$ of weight $k$ with integral Fourier coefficients and constant term equal to 1 . Then $g=G_{k}+\frac{B_{k}}{2 k} h$ is a cusp form congruent to $G_{k}$ modulo $b_{k}$.

Let $K_{k}$ denote the algebraic number field generated over the field of rational numbers $\mathbb{Q}$ by all Fourier coefficients of all primitive forms of weight $k$, and let $O_{k}$ be the ring of algebraic integers in $K_{k}$.

Let $p$ be a prime dividing $b_{k}$ and $\mathfrak{P}$ a prime ideal of $O_{k}$ dividing $p$. Proposition 1 implies that $g$ is a Hecke eigenform with eigenvalues $\sigma_{k-1}(n)$ modulo $\mathfrak{P}$. By Lemma 6.11 of [3] there exists a primitive Hecke eigenform $f$ whose eigenvalues are congruent to $\sigma_{k-1}(n)$ modulo $\mathfrak{P}$. Since eigenvalues of Hecke operators happen to be the Fourier coefficients of a primitive eigenform, we have

Proposition 2. Let $\mathfrak{P}$ be a prime divisor of $b_{k}$ in $K_{k}$. Then there exists a primitive Hecke eigenform $f$ such that $f \equiv G_{k} \bmod \mathfrak{P}$.

R e m ark. It was recently communicated to the author that it was Prof. K. Doi who first found the congruences of the type described in the above propositions.

In what follows we will need the following condition.

$\mathrm{H} 1$. The primitive form which is congruent to $G_{k}$ modulo $\mathfrak{P}$ is unique.

It is evident that this condition is true when $\operatorname{dim} S_{k}=1$ and it is easy to check by direct computer calculation that it is true when $\operatorname{dim} S_{k}=2$. The following assertion shows that one can expect it to be true under some additional conditions.

Proposition 3. Consider a prime $p \mid b_{k}$ such that $p>t\left(2^{k-1}+1+2^{k / 2}+\right.$ $2^{(k-2) / 2}$ ), where $t=\operatorname{dim} S_{k}$. Let $\mathfrak{P}$ be a prime divisor of $p$ in $K_{k}$. Then it cannot be true that $f \equiv G_{k}$ mod $\mathfrak{P}$ for all normalised Hecke eigenforms $f$ in $S_{k}$. 
The absolute value of the second Fourier coefficient of a primitive form in $S_{k}$ is less than or equal to $2^{k / 2}+2^{(k-2) / 2}$. One can find the surprisingly simple proof of this estimate in [8], where a much more general setting was considered.

To prove our proposition we consider the sum of all the primitive forms. It is a modular form $F$ with rational integral Fourier coefficients. If the conclusion of the proposition is false, then $F \equiv t G_{k} \bmod \mathfrak{P}$. It yields $F \equiv$ $t G_{k} \bmod p$. Consider the second Fourier coefficients in this congruence. They are not equal. Moreover, $p$ is greater than the sum of their absolute values. Therefore they cannot be congruent modulo $p$, and our proposition is proved.

Proposition 3 proves $\mathrm{H} 1$ in the case when $t$ is prime and the Galois group $\operatorname{Gal}\left(K_{k} / \mathbb{Q}\right)$ acts on primitive forms transitively. In this case if any two primitive forms are congruent modulo $\mathfrak{P}$ they all would be. Conjecturally, this Galois group almost always acts on primitive forms transitively.

As is well known, the set of positive integers which have a prime divisor greater than their $\alpha$ th power has a positive density $\delta(\alpha)$ which tends to 1 as $\alpha$ tends to 0 . Since $\left|B_{k}\right|>(k /(2 \pi e))^{k}$ and $t \leq k / 12$, it becomes plausible that $B_{k}$ has a prime factor $p>t\left(2^{k-1}+1+2^{k / 2}+2^{(k-2) / 2}\right)$ for most large values of $k$.

We can now state our main result.

Theorem 1. Let $k \geq 12$ be an even integer. Consider a triple $(\xi, \chi, l)$ which consists of primitive Dirichlet characters $\xi$ modulo $B$ and $\chi$ modulo $M$, and an integer $l$ satisfying the following conditions:

$$
B M \text { is square-free, } \quad \xi \chi(-1)=(-1)^{l}, \quad \text { and } \quad 1 \leq l \leq k-1 .
$$

Denote by $\Omega$ the Galois extension of $K_{k}$ obtained by adjoining the values of the Gauss sums $G(\chi)$ and $G(\xi)$ and the characters $\chi, \xi$. Let $O$ be the ring of integers in $\Omega$. Let $p$ be a prime divisor of $b_{k}$, and let $\mathfrak{P}$ be a prime ideal of $O_{k}$ dividing $p$ that satisfies the condition $\mathrm{H} 1$ and $\mathfrak{P}^{\prime}$ a prime ideal in $O$ dividing $\mathfrak{P}$. Let $f$ be the unique cusp form of weight $k$ which is congruent to the Eisenstein series $G_{k}$ modulo $\mathfrak{P}$. Put

$$
\begin{aligned}
\kappa & =\kappa(k, l, \chi, \xi) \\
& = \begin{cases}G(\chi) L(1+l-k, \chi) L(1-l, \chi) & \text { if } \xi \text { is trivial, } \\
G(\xi) L(0, \xi) L(2-k, \xi) & \text { if } \chi \text { is trivial and } l=1, \\
-G(\xi) L(0, \xi) L(2-k, \bar{\xi}) & \text { if } \chi \text { is trivial and } l=k-1, \\
0 & \text { otherwise. }\end{cases}
\end{aligned}
$$

Suppose that the numbers $L(1+l-k, \xi \chi), L(1-l, \bar{\xi} \chi), L(1-l, \xi \bar{\chi})$ are $\mathfrak{P}^{\prime}$ integral. Then the number $c_{k-l}^{-1} \Omega_{f}^{-1} L_{f}(k-1, \xi) L_{f}(k-l, \chi)$ is algebraic and there is a number $C(f) \in K_{k}$ which depends only on $f$ such that

(1) $\quad(-M)^{k-l} B^{k-1} C(f) c_{k-l}^{-1} \Omega_{f}^{-1} L_{f}(k-1, \xi) L_{f}(k-l, \chi) \equiv-\kappa \bmod \mathfrak{P}^{\prime}$, 
where

$$
c_{h}=\frac{(2 \pi i)^{h}}{(h-1) !}, \quad \Omega_{f}=\frac{2^{2 k-3} \pi^{k-1}}{(k-2) !}\langle f, f\rangle .
$$

The brackets $\langle\cdot, \cdot\rangle$ denote as usual the Petersson scalar product.

Since for $n \geq 1, L(1-n, \chi)=-B_{n, \chi} / n$, one can reformulate the conditions of the theorem in terms of the generalised Bernoulli numbers. Their definition

$$
\sum_{1 \leq a \leq m} \frac{\chi(a) t e^{a t}}{e^{m t}-1}=\sum_{n \geq 0} B_{n, \chi} \frac{t^{n}}{n !}
$$

yields that $\kappa$ is an algebraic number.

Before giving the proof of this theorem we are going to derive some corollaries. The transcendental quantities in the left-hand side of (1) depend on $f$. Their dependence on $l$ is hidden in $c_{k-l}$. It can be easily taken under control. The number $C(f)$ is defined by (11) below. One can consider these numbers as normalisation constants (connected with $f$ ). We will eliminate them by dividing (1) by itself. This procedure eliminates the dependence on the data $(\xi, \chi, l)$.

Till the end of the section we fix $\mathfrak{P}|p| b_{k}$ and assume H1. We also fix the unique cusp form $f$ of weight $k \geq 12$ such that $f \equiv G_{k}$ mod $\mathfrak{P}$. Consider two triples $\left(\xi_{i}, \chi_{i}, l_{i}\right), i=1,2$. In order to simplify the final formulae we are going to fix some data in these triples. The most natural way is to put $\xi_{1}=\xi_{2}=\psi^{0}$ (the trivial character). This gives the following:

Corollary 1. Assume $p>k$. Let $\chi_{i}, i=1,2$, be two primitive Dirichlet characters with square-free conductors $M_{i}$. Let $l_{i}$ be two integers $1 \leq l_{i} \leq$ $k-1$ such that $\chi_{i}(-1)=(-1)^{l_{i}}$. Let $\mathfrak{P}^{\prime}$ be a prime divisor of $\mathfrak{P}$ in $\Omega$. We suppose that $\mathfrak{P}^{\prime}$ does not divide $L\left(1+l_{2}-k, \chi_{2}\right), L\left(1-l_{2}, \chi_{2}\right), M_{2}$ and the numbers $L\left(1+l_{i}-k, \chi_{i}\right), L\left(1-l_{i}, \chi_{i}\right)$ and $L\left(1-l_{i}, \bar{\chi}_{i}\right)$ are $\mathfrak{P}^{\prime}$-integral. Then the following congruence holds:

$$
\begin{aligned}
& (-2 \pi i)^{l_{1}-l_{2}} \frac{L_{f}\left(k-l_{1}, \chi_{1}\right)}{L_{f}\left(k-l_{2}, \chi_{2}\right)} \\
& \quad \equiv \frac{G\left(\chi_{1}\right)}{G\left(\chi_{2}\right)} \cdot \frac{\left(k-l_{2}-1\right) ! M_{1}^{l_{1}}}{\left(k-l_{1}-1\right) ! M_{2}^{l_{2}}} \cdot \frac{L\left(1+l_{1}-k, \chi_{1}\right) L\left(1-l_{1}, \chi_{1}\right)}{L\left(1+l_{2}-k, \chi_{2}\right) L\left(1-l_{2}, \chi_{2}\right)} \bmod \mathfrak{P}^{\prime}
\end{aligned}
$$

Note that the denominator on the left-hand side is non-zero since the assumptions yield $\kappa\left(k, l_{2}, \chi_{2}, \xi_{2}\right) \not \equiv 0 \bmod \mathfrak{P}^{\prime}$.

As was mentioned in the introduction, one can explain this result using the (slightly modified) heuristic argument from [6]. In accordance with that philosophy we once more encounter the situation when Ramanujan type congruences hold after a transcendental procedure of obtaining special values and removing some transcendental factors.

It is worthwhile to note that Corollary 1 contains plenty of congruences. 
Now we are going to show how one can derive as special cases of our theorem two congruences proved in [6] and [7] using different methods.

Put $l_{1}=l_{2}=k / 2$ with $2 \mid l_{i}$ in Corollary 1 . Take $\chi=\chi_{1}$ to be the character of a real quadratic field with discriminant $M$ and $\chi_{2}$ the identity character. After noticing that $G(\chi)=M^{1 / 2}$ we obtain for $\mathfrak{P}$ that divides $p$, $p>k, p$ does not divide $M, b_{k / 2}$ and the denominator of $\left(B_{k / 2, \chi}\right)$ :

$$
M^{(1-k) / 2} \frac{L_{f}(k / 2, \chi)}{L_{f}(k / 2)} \equiv \frac{L(1-k / 2, \chi)^{2}}{\zeta(1-k / 2)^{2}} \bmod \mathfrak{P} .
$$

The last formula for $k=12$ is precisely that of Corollary to Theorem 1 of [7]. We can also take any other $k$ divisible by 4 to obtain other congruences of this type.

In the spirit of [7] we can also consider (2) as a congruence between the squares of Fourier coefficients of the cusp form of half integer weight which corresponds to $f$ via the Shimura map, and the Eisenstein series of half integer weight discovered by Cohen [1]. From this point of view our Corollary 1 can serve as a partial answer to the Remark in [7] since it gives a more conceptual way to prove such congruences. A more detailed discussion of this subject is contained in [2].

Let us now return to Theorem 1 and put $l_{2}=k-2$. Set $l_{1}=k-m-1$, where $m$ is odd and $\chi_{1}=\chi_{2}=\xi_{1}=\xi_{2}=\psi^{0}$. We suppose that the rational prime $p>k$ does not divide $b_{k-2}$ and the denominators of $\zeta\left(1+l_{1}-k\right)$ and $\zeta\left(1-l_{1}\right)$. Substituting all this data into (1) and taking into account the definition of the periods of a cusp form $f$ (cf. [6])

$$
r_{m}(f)=\frac{m ! i^{m+1}}{(2 \pi)^{m+1}} L_{f}(m+1), \quad 0 \leq m \leq k-2,
$$

we obtain congruences

$$
\frac{r_{m}(f)}{r_{1}(f)} \equiv \frac{\zeta(-m) \zeta(m-k+2)}{\zeta(-1) \zeta(3-k)} \bmod \mathfrak{P} .
$$

For $k$ such that $\operatorname{dim} S_{k}=1,(3)$ is identical to the assertion of [6], Theorem 2.

Now put $\chi_{1}=\chi_{2}=\psi^{0}, l_{2}=1$, and take $l_{1}$ to be an odd number not equal to 1 nor $k-1$. A direct computer calculation shows that for every $k$ such that $\operatorname{dim} S_{k} \leq 2$ and each $\mathfrak{P}$ dividing $b_{k}$ we can easily choose an odd quadratic Dirichlet character $\xi$ such that $\mathfrak{P}$ does not divide the numbers $L(2-k, \xi)$ and $L(0, \xi)$. It is natural to conjecture that such a character exists for every weight $k$. Taking $\xi_{1}=\xi_{2}=\xi$ we obtain

$$
(-2 \pi i)^{l_{1}-1} \frac{L_{f}\left(k-l_{1}\right)}{L_{f}(k-1)} \equiv 0 \bmod \mathfrak{P} .
$$

In terms of periods of cusp forms we obtain the following 
COROLlary 2. Suppose that there exists a quadratic primitive Dirichlet character $\xi$ modulo $B$ such that $\mathfrak{P}$ does not divide the numbers $L(2-k, \xi)$ and $L(0, \xi)$. Then for each even $m$ satisfying $2 \leq m \leq k-2$,

$$
\frac{r_{m}(f)}{r_{0}(f)} \equiv 0 \bmod \mathfrak{P} \text {. }
$$

2. In this section we prove the theorem formulated in the previous section. Put

$$
\Lambda(k, l, \xi, \chi)=\frac{2^{3 k-l-3} \pi^{2 k-l-1} i^{3 k-3 l}}{(k-2) !(k-l-1) !} M^{-k} B^{1-k} .
$$

Using Rankin's method we are going to construct a modular form $F_{l, \xi, \chi}$ such that

$$
L_{f}(k-1, \xi) L_{f}(k-l, \chi)=\Lambda(k, l, \xi, \chi)\left\langle f, F_{l, \xi, \chi}\right\rangle .
$$

The brackets $\langle\cdot, \cdot\rangle$ denote the Petersson scalar product which we define in general for two modular forms $f$ and $h$ of weight $k$ on a congruence subgroup $\Gamma_{1}(N)$ when $f h$ is a cusp form:

$$
\langle f, g\rangle=\int_{H \backslash \Gamma_{1}(N)} \overline{f(\tau)} h(\tau) y^{k-2} d x d y, \quad \tau=x+i y .
$$

After an explicit calculation of the Fourier coefficients of the modular function $F_{l, \xi, \chi}$ the proof reduces to a problem in linear algebra. The results of this calculation are stated in the following:

Proposition 4. Suppose that two primitive Dirichlet characters $\xi$ modulo $B$ and $\chi$ modulo $M$ are such that $B M$ is square-free and $\xi \chi(-1)=(-1)^{l}$ where $1 \leq l \leq k-1$. Let $\kappa_{l, \xi, \chi}(n)$ be the nth Fourier coefficient of the modular form $F_{l, \xi, \chi}$ defined by (4). Then

$$
\kappa_{l, \xi, \chi}(0)=M^{l} \kappa(k, l, \chi, \xi) \quad(\text { defined in Theorem } 1)
$$

and for $n>0$,

$$
\kappa_{l, \xi, \chi}(n)=2 G(\chi) G(\xi)\left(Q+R+R_{1}+S+S_{1}\right)
$$

where

$$
\begin{aligned}
Q= & 2 \sum_{x|B, y| M}(B / x)^{l-1} \xi_{1}(-1) \xi(M / y) \chi(B / x) \\
& \times \sum_{r_{1}+r_{2}=n x y} \sigma_{k-l-1}\left(\bar{\chi}_{2} \bar{\xi}_{2}, \chi_{1} \xi_{1}, r_{1}\right) \sigma_{l-1}\left(\chi_{2} \bar{\xi}_{1}, \bar{\chi}_{1} \xi_{2}, r_{2}\right), \\
R= & L(1+l-k, \chi \xi) B^{l-1} \xi(M) \chi(B) \sigma_{l-1}(\chi, \xi, n), \\
R_{1}= & \begin{cases}M^{l-1} L(0, \chi \xi) \xi(-1) \bar{\chi}(B) \sigma_{l-1}(\bar{\xi}, \bar{\chi}, n) & \text { if } l=k-1, \\
0 & \text { otherwise, }\end{cases}
\end{aligned}
$$

and 


$$
\begin{aligned}
S & =B^{k-l-1} \xi(-M) \bar{\chi}(B) L(1-l, \bar{\xi} \chi) \sigma_{k-l-1}(\bar{\chi}, \xi, n), \\
S_{1} & = \begin{cases}B^{l-1} M^{k-l-1} L(0, \bar{\xi} \chi) \chi(B) \bar{\xi}(M) L(1-l, \xi \bar{\chi}) \sigma_{k-l-1}(\bar{\xi}, \chi, n) \\
\text { if } l=1,\end{cases}
\end{aligned}
$$

Here for each $x \mid B$ and $y \mid M$ we denoted by $\xi_{i}$ and $\chi_{i}, i=1,2$, the characters modulo $x, B / x, y$ and $M / y$ respectively such that $\xi=\xi_{1} \xi_{2}$ and $\chi=\chi_{1} \chi_{2}$.

Consider a triple $(l, \xi, \chi)$ satisfying the conditions of Theorem 1 . We write simply $F$ and $\kappa(n)$ instead of $F_{l, \xi, \chi}$ and $\kappa_{l, \xi, \chi}(n)$ for this triple.

In the following we will not need the explicit formulae for $\kappa(n), n \geq 1$. We only claim that they belong to $O$ under the conditions of Theorem 1 .

Let us for a moment assume this proposition to be true and finish the proof of Theorem 1. After that we will prove this proposition.

Let $f=f_{1}, \ldots, f_{t}$, where $f_{i}=\sum_{n>0} a_{i}(n) q^{n}$, be the set of primitive forms of weight $k$. Since these forms form a basis of the $\mathbb{C}$-linear space $S_{k}$, for an arbitrary modular form $F$ of weight $k$ one has

$$
F=\alpha_{0} G_{k}+\alpha_{1} f_{1}+\ldots+\alpha_{t} f_{t} .
$$

Then

$$
\alpha_{1}=\frac{\langle f, F\rangle}{\langle f, f\rangle} .
$$

Since we consider modular forms on the full modular group, the correspondence which sends a modular form $g=\sum_{n>0} a(n) q^{n}$ of weight $k$ to the $(t+1)$-vector $(a(0), \ldots, a(t))$ is one-to-one and preserves integrality and congruences. By Cramer's method

$$
\alpha_{1}=\frac{\left|G_{k}, F, f_{2}, \ldots, f_{t}\right|}{\left|G_{k}, f, f_{2}, \ldots, f_{t}\right|} .
$$

Here

$$
\left|\varphi_{1}, \ldots, \varphi_{l}\right|=\left|\begin{array}{ccc}
c_{1}(0) & \ldots & c_{l}(0) \\
\ldots & \ldots & \ldots \\
c_{1}(l-1) & \ldots & c_{l}(l-1)
\end{array}\right|, \quad \varphi_{i}=\sum_{n \geq 0} c_{i}(n) q^{n} .
$$

Combining (5) with (4) we obtain

$$
L_{f}(k-1, \xi) L_{f}(k-l, \chi)=\Lambda(k, l, \xi, \chi)\langle f, f\rangle \frac{\left|G_{k}, F_{l, \xi, \chi}, f_{2}, \ldots, f_{t}\right|}{\left|G_{k}, f, f_{2}, \ldots, f_{t}\right|} .
$$

The determinant in the numerator on the right-hand side has only two non-zero entries in its first row. We expand it through this row. Then

$$
\left|G_{k}, F, f_{2}, \ldots, f_{t}\right|=-\frac{B_{k}}{2 k}\left|F, f_{2}, \ldots, f_{t}\right|^{+}-\kappa(0)\left|G_{k}, f_{2}, \ldots, f_{t}\right|^{+},
$$


where

$$
\left|\varphi_{1}, \ldots, \varphi_{l}\right|^{+}=\left|\begin{array}{ccc}
c_{1}(1) & \ldots & c_{l}(1) \\
\ldots & \ldots & \ldots \\
c_{1}(l) & \ldots & c_{l}(l)
\end{array}\right|
$$

We claim that there do not exist numbers $\beta_{0}, \beta_{2}, \ldots, \beta_{t} \in \Omega$ such that $\mathfrak{P}^{\prime}$ does not divide $\beta_{0}$ and

$$
\sum_{2 \leq i \leq t} \beta_{i} f_{i} \equiv \beta_{0} G_{k} \bmod \mathfrak{P}^{\prime}
$$

Otherwise one can suppose that the set of eigenforms $\left\{f_{i}\right\}$ for which there exist $\beta_{i}$ and $\beta_{0}$ as above is minimal. Suppose that $f_{m}$ for some $2 \leq m \leq t$ belongs to this set. Applying the Hecke operator $T_{n}$ to (8) and eliminating the eigenform $f_{m}$ we obtain

$$
\sum_{\substack{2 \leq i \leq t \\ i \neq m}} \beta_{i}\left(a_{i}(n)-a_{m}(n)\right) f_{i} \equiv \beta_{0}\left(\sigma_{k-1}(n)-a_{m}(n)\right) G_{k} \bmod \mathfrak{P}^{\prime}
$$

This contradicts minimality of the set of eigenforms $\left\{f_{i}\right\}$ unless for all $n \geq 1$ one has $\sigma_{k-1}(n) \equiv a_{m}(n) \bmod \mathfrak{P}^{\prime}$. This implies that, for some $2 \leq m \leq t$, $f_{m} \equiv G_{k} \bmod \mathfrak{P}^{\prime}$. Since the Fourier coefficients of $f_{m}$ belong to $K_{k}$, this yields $f_{m} \equiv G_{k} \bmod \mathfrak{P}$, which contradicts H1. Note that this argument is similar to Lemma 6.11 of [3].

Let us prove that $\left|G_{k}, f_{2}, \ldots, f_{t}\right|^{+} \neq 0$. Otherwise the columns of the determinant are linearly dependent. Since the vectors $\left(a_{i}(0), \ldots, a_{i}(t)\right), i=$ $2, \ldots, t$, are linearly independent, and $a_{i}(0)=0$ for all $i \geq 1$, the first coefficient of this dependence is non-zero. Since $\mathfrak{P}$ divides $B_{k} /(2 k)$ this yields (8).

Let us consider the system of $t$ linear equations:

$$
\kappa(1)=\alpha_{0} \sigma_{k-1}(1)+\alpha_{2} a_{2}(1)+\ldots+\alpha_{t} a_{t}(1),
$$

$$
\kappa(t)=\alpha_{0} \sigma_{k-1}(t)+\alpha_{2} a_{2}(t)+\ldots+\alpha_{t} a_{t}(t) .
$$

Since the determinant of (9) is non-zero, the system has a unique solution, and one can apply Cramer's method. Let $R=(O)_{\mathfrak{P}^{\prime}}$. We claim that

$$
\alpha_{0}=\frac{\left|F, f_{2}, \ldots, f_{t}\right|^{+}}{\left|G_{k}, f_{2}, \ldots, f_{t}\right|^{+}} \in R .
$$

Otherwise since $\kappa \in R$ one can obtain (8) by multiplying (9) by $\alpha_{0}^{-1}$.

Put

$$
C(f)=\frac{\left|G_{k}, f, f_{2}, \ldots, f_{t}\right|}{\left|G_{k}, f_{2}, \ldots, f_{t}\right|^{+}}
$$


Combining (6) and (7) we get

$$
(-M)^{k-l} B^{k-1} c_{k-l}^{-1} \Omega_{f}^{-1} C(f) L_{f}(k-1, \xi) L_{f}(k-l, \chi)=-b_{k} \alpha_{0}-\kappa(0),
$$

and (10) now yields the congruence of Theorem 1.

We now turn to the proof of Proposition 4. We first state a proposition which is in fact a variation of the classical Rankin's method.

Let us define the numbers $b(n)$ for $n \geq 1$ by

$$
L(s, \xi) L(s+1-l, \chi)=\sum_{n \geq 1} b(n) n^{-s} .
$$

It is known (see [11], pp. 798-799), that if $l \neq 2$ then there exists a unique modular form $g$ of weight $l$ on the congruence subgroup $\Gamma_{0}(B M) \subset \mathrm{SL}(2, \mathbb{Z})$ with Dirichlet character $\chi \xi$ such that

$$
g(z)=\sum_{n \geq 0} b(n) q^{n}
$$

In the following we will use a suitable notation

$$
(a z+b)^{x}|a z+b|^{y}=(a z+b)^{x+|y|} .
$$

Let us define an Eisenstein series

$$
E(z)=\left.\sum_{(m, n)} \frac{\chi \xi(n)}{(m B M z+n)^{k-l+|2 s|}}\right|_{s=0}
$$

where the sum on the right is over all pairs of integers $(m, n)$ except $(m, n)=$ $(0,0)$. The following proposition is a restatement of Theorem 2 of [11].

Proposition 5. Let $f=\sum_{n>1} a(n) q^{n}$ be a primitive form of weight $k$ on $\operatorname{SL}(2, \mathbb{Z})$. Let $\chi, \xi$ and $l$ be as above. Then

$$
L_{f}(k-1, \xi) L_{f}(k-l, \chi)=\frac{2^{2 k-3} \pi^{k-1}}{(k-2) !}\left\langle f, \operatorname{Tr}_{\mathrm{SL}(2, \mathbb{Z})}^{\Gamma_{0}(B M)}(g E)\right\rangle .
$$

In what follows we calculate the Fourier coefficients of the modular form $\operatorname{Tr}_{\mathrm{SL}(2, \mathbb{Z})}^{\Gamma_{0}(B M)}(g E)$ explicitly. It is a special case of an analogous but more general calculation given in [4].

In order to calculate the action of the trace operator $\operatorname{Tr}_{\mathrm{SL}(2, \mathbb{Z})}^{\Gamma_{0}(B M)}$ from the space of $\Gamma_{0}(B M)$-modular forms into the space of $\operatorname{SL}(2, \mathbb{Z})$-modular forms we fix the following system of $\Gamma_{0}(B M)$-right coset representatives in $\mathrm{SL}(2, \mathbb{Z})$. For each $x \mid B$ and $y \mid M$ we choose a matrix $\left(\begin{array}{ll}a & b \\ c & d\end{array}\right) \in \mathrm{SL}(2, \mathbb{Z})$ such that $\operatorname{gcd}(c, B M)=B M /(x y)$ and $c^{*} d$ runs through all residues modulo $x y$. Here $c^{*}$ denotes an integer satisfying the condition $c^{*} c \equiv 1 \bmod x y$. The assertion that the described set of matrices is in fact a set of right coset representatives is contained in [4] and can be easily checked. 
We are now going to apply the matrices of this set to the function $g E$ and to compute the Fourier expansion of the obtained functions. Since both $g$ and $E$ are Eisenstein series, we will need the following proposition on the Fourier expansion of Eisenstein series.

Proposition 6. Let $N$ be a natural number. For a function $\phi$ on $\mathbb{Z} / N \mathbb{Z} \times$ $\mathbb{Z} / N \mathbb{Z}$ define its partial Fourier transform $P \phi$ on $\mathbb{Z} / N \mathbb{Z} \times \mathbb{Z} / N \mathbb{Z}$ by the formula

$$
P \phi(n, m)=\sum_{a \bmod N} \phi(a, m) \exp (2 \pi i a n / N) .
$$

Define an Eisenstein series by

$$
E_{k, \phi}(z)=\left.\sum_{m, n} \frac{\phi(n, m)}{(m z+n)^{k+|2 s|}}\right|_{s=0} .
$$

If the function $\phi$ is not identically equal to one, then one has the following Fourier expansion:

$$
\begin{aligned}
& N^{k}(-2 \pi i)^{-k}(k-1) ! E_{k, \phi}(N z) \\
& \quad=L(1-k, P \phi(\cdot, 0))+A_{1} \sum_{d d^{\prime}>0} \operatorname{sgn}(d) d^{k-1} P \phi\left(d, d^{\prime}\right) q^{d d^{\prime}} .
\end{aligned}
$$

Here

$$
A_{1}= \begin{cases}L(0, P \phi(0, \cdot)-P \phi(0,-\cdot)) / 2 & \text { if } k=1, \\ 0 & \text { otherwise. }\end{cases}
$$

The proof of this proposition is contained in [5], Lemmas 3.2.5 and 3.4.1.

We proceed with the calculation of the action of the trace operator. For each $x \mid B$ and $y \mid M$ let us introduce the following Eisenstein series:

$$
\begin{gathered}
E^{x y}(z)=\left.\sum_{m, n} \frac{\chi_{1}(m) \xi_{1}(m) \chi_{2}(n) \xi_{2}(n)}{(m(M B /(x y)) z+n)^{k-l+|2 s|}}\right|_{s=0}, \\
g^{x y}(z)=\left.\lambda \sum_{m, n} \frac{\xi_{1}(n) \xi_{2}(m) \bar{\chi}_{1}(m) \bar{\chi}_{2}(n)}{(m(x M / y) z+n)^{l+|2 s|}}\right|_{s=0}
\end{gathered}
$$

where

$$
\lambda=G(\chi) \frac{(l-1) ! M^{l-1}}{2(-2 \pi i)^{l}} .
$$

It is evident that in accordance with our previous notations, $E^{1}(z)=E(z)$. Using Proposition 6 and definition (12) one obtains $g^{1}(z)=g(z)$.

A direct calculation shows that if a matrix $\left(\begin{array}{ll}a & b \\ c & d\end{array}\right)$ belongs to the system of $\Gamma_{0}(B M)$-right representatives which was fixed above with $\operatorname{gcd}(c, B M)=$ $B M /(x y)$, then

$$
\left.E\right|_{k-l}\left(\begin{array}{ll}
a & b \\
c & d
\end{array}\right)=\chi_{1} \xi_{1}\left(c^{*} B M /(x y)\right) \chi_{2} \xi_{2}(x y) \bar{\chi}_{2} \bar{\xi}_{2}(d)(x y)^{l-k} E^{x y}\left(\frac{z+c^{*} d}{x y}\right)
$$


and

$$
\begin{aligned}
& \left.g\right|_{l}\left(\begin{array}{ll}
a & b \\
c & d
\end{array}\right) \\
& \quad=\xi_{1}(-c y) \xi_{2}(d M / y) \bar{\chi}_{1}\left(-c^{*} M / y\right) \bar{\chi}_{2}(-y) \chi_{2}(d) \bar{\xi}(M) y^{-l} g^{x y}\left(\frac{z+c^{*} d}{x y}\right) .
\end{aligned}
$$

It follows that

$$
\begin{aligned}
\operatorname{Tr}_{\mathrm{SL}(2, \mathbb{Z})}^{\Gamma_{0}(B M)}(g E) & \\
= & \left.\sum_{x|B, y| M} \sum_{c^{*} d \bmod x y}(g E)\right|_{k}\left(\begin{array}{cc}
a & b \\
c & d
\end{array}\right) \\
& =\lambda \sum_{x|B, y| M} x^{l-k+1} y^{-k+1} \xi(x-B / x) \chi(M / y-y)\left(g^{x y} E^{x y}\right) \mid U_{x y}
\end{aligned}
$$

where we denoted by $U_{x y}$ the operator acting on Fourier series as

$$
U_{x y}: \sum_{n \geq 0} c(n) q^{n} \mapsto \sum_{n \geq 0} c(x y n) q^{n} .
$$

In order to finish the proof of Proposition 4 it remains to apply Proposition 6 to the Eisenstein series on the right-hand side of (14). Theorem 1 is now proved.

\section{References}

[1] H. Cohen, Sums involving the values at negative integers of $L$-functions of quadratic characters, Math. Ann. 217 (1975), 271-285.

[2] B. Datskovsky and P. Guerzhoy, On Ramanujan congruences for modular forms of higher weight, Proc. Amer. Math. Soc., to appear.

[3] P. Deligne and J.-P. Serre, Formes modulaires de poids 1, Ann. Sci. Ecole Norm. Sup. 7 (1974), 507-530.

[4] P. I. Gerzhoŭ and A. A. Panchishkin, Finiteness criterion for the number of rational points on twisted Weil elliptic curves, Zap. Nauch. Sem. LOMI 160 (1987), $41-53$.

[5] N. M. Katz, p-adic continuation of real analytic Eisenstein series, Ann. of Math. 104 (1976), 459-571.

[6] N. Koblitz, Congruences for periods of modular forms, Duke Math. J. 54 (1987), 361-373.

[7] —, p-adic congruences and modular forms of half integer weight, Math. Ann. 274 (1986), 199-220.

[8] W. Kohnen, A simple remark on eigenvalues of Hecke operators on Siegel modular forms, Abh. Math. Sem. Univ. Hamburg 57 (1987), 33-36.

[9] Yu. I. Manin, Periods of parabolic forms and p-adic Hecke series, Mat. Sb. 92 (1973), 378-401 (in Russian). 
[10] K. A. Ribet, Congruence relations between modular forms, in: Proceedings of the International Congress of Mathematicians (Warsaw, 1983), PWN, Warszawa, 1984, 503-514.

[11] G. Shimura, The special values of the zeta-functions associated with cusp forms, Comm. Pure Appl. Math. 29 (1976), 783-804.

[12] H. P. F. Swinnerton-Dyer, On l-adic representations and congruences for coeffcients of modular forms, in: Modular Functions of One Variable III, Lecture Notes in Math. 350, Springer, 1973, 1-55.

[13] - Congruence properties of $\tau(n)$, in: Ramanujan Revisited, Proceedings of the Centenary Conference, Academic Press, 1988, 289-311.

Fakultät für Mathematik und Informatik

Universität Mannheim

Seminargebäude A5

D-68131 Mannheim, Germany

E-mail: pasha@euklid.math.uni-mannheim.de

pasha@techunix.technibn.ac.il

Received on 10.7.1995

and in revised form on 4.2.1997 